\title{
COMPARAÇÃO ENTRE PRAZIQUANTEL E OXAMNIQUINE NO TRATAMENTO DA ESQUISTOSSOMOSE MANSONI
}

\author{
Antônio Emanuel e Aluízio Prata
}

\begin{abstract}
Tratamos 120 pacientes masculinos com idade de 17 a 19 anos, portadores de esquistossomose mansoni nas formas intestinal e hepatointestinal, pareados de acordo com o numero de ovos por grama de fezes. Um membro de cada par tomou oxamniquine na dose de $15 \mathrm{mg} / \mathrm{kg}$ e o outro, praziquantel na dose de $55 \mathrm{mg} / \mathrm{kg}$, em cápsulas.

Dos 106 pacientes avaliados com pelo menos dois exames de fezes, negativaram-se 44 (83\%) dos que tomaram praziquantel e 41 (77,3\%) dos que tomaram oxamniquine.

Ambas as drogas foram relativamente bem toleradas, com ligeira vantagem da oxamniquine.
\end{abstract}

Palavras chaves: Esquistossomose mansoni. Terapêtica. Oxamniquine. Praziquantel.

A oxamniquine vem sendo usada largamente no tratamento da esquistossomose 2581214 . No Brasil ela tem sido a droga de escolha inclusive para programas de tratamento em massa ${ }^{4}{ }^{11}$. O praziquantel foi mais recentemente introduzido na terapêutica da esquistossomose ${ }^{3} \quad 913$.

Nosso trabalho tem por objetivo comparar as duas drogas no tratamento da esquistossomose, quanto à eficácia e à tolerabilidade.

\section{MATERIAL E MÉTODOS}

Tratamos 120 pacientes masculinos, com idade de 17 a 19 anos, com esquistossomose mansoni nas formas intestinal e hepatointestinal, todos alunos da Escolà de Aprendizes e Marinheiros de Olinda - PE. Eles foram submetidos a dois exames quantitativos de fezes, pelo método de Kato, moficado por Katz e cols ${ }^{7}$ e os resultados variaram de 12 a 1.536 ovos de Shistosoma mansoni por grama de fezes, com a média de 252,5.

Os pacientes foram pareados pelo número de ovos por grama de fezes. Vinte e seis pares tinham exatamente o mesmo número de ovos e os outros

Núcleo de Medicina Tropical e Nutrição, Universidade de Brasília, CEP 70909 Brasília, DF, Brasil

Recebido para publicação em 31-12-82
27 pares apresentavam diferença mínima. Sete pares não tiveram controle de cura.

Um membro de cada par foi tratado com oxamniquine e o outro com praziquantel. A oxamniquine foi administrada na dose de $15 \mathrm{mg} /$ quilo de peso corporal e o praziquantel na de 55mg. Ambos foram dados em cápsulas de desintegração entérica, em dose única, de forma duplo-cega e sempre à nossa vista. Em seguida, os pacientes eram liberados para suas atividades normais. No dia seguinte todos foram reexaminados para se avaliar a tolerância aos medicamentos, com base nas informações prestadas por cada paciente de forma espontânea, isto é, sem ser em resposta a um questionário.

Tabela 1 - Número de exames de fezes controles realizados em 106 pacientes com esquistossomose tratados com praziquantel ou oxamniquine

\begin{tabular}{lcrc}
\hline \multirow{2}{*}{ Medicamento } & \multicolumn{3}{c}{ Exames de Fezes } \\
\cline { 2 - 4 } & 2 & 3 & 4 \\
\hline Praziquantel & 18 & 10 & 25 \\
Oxamniquine & 18 & 3 & 32 \\
\hline Total & 36 & 13 & 57 \\
\hline
\end{tabular}


Emanuel A, Prata A. Comparação entre praziquantel e oxamniquine no tratamento da esquistossomose mansoni. Revista da Sociedade Brasileira de Medicina Tropical 16:90-93, Abr-Jun, 1983

$O$ controle de cura constou de exames de fezes pela mesma técnica, realizado a partir do $40^{\circ}$ dia após o tratamento. O tempo de seguimento foi, no máximo, de dez meses e, neste período, os pacientes permaneceram na Escola e, portanto, ao abrigo de reinfecção.

Os pacientes fizeram de dois a quatro exames de fezes de controle (Tabela 1). Cada exame de controle consistiu na análise de duas lâminas examinadas a partir da mesma amostra de fezes. Consideramos não curado todo paciente que apresentasse um ou mais ovos em qualquer dos exames de fezes realizados durante o periodo de controle.

\section{RESULTADOS}

Dos 120 pacientes tratados, em apenas $106 \mathrm{pu}$ demos avaliar o par adequadamente, quanto à eficácia terapêutica. Destes, $41(77,3 \%)$ tratados com oxamniquine e $44(83 \%)$ tratados com praziquan- tel mostraram todos os exames de fezes negativos e foram considerados curados (Tabela 2).

Tabela 2 - Resultados do tratamento de 106 pacientes com esquistossomose tratados com praziquantel ou oxamniquine

\begin{tabular}{lcc}
\hline Medicamento & Controlados & Curados \\
\hline Praziquantel & 53 & $44(83,0 \%)$ \\
Oxamniquine & 53 & $41(77,3 \%)$ \\
\hline
\end{tabular}

Nos noves pacientes que tomaram praziquantel e não foram curados houve redução da carga parasitária, em média de 281 para 14 ovos por grama de fezes $(95,1 \%)$. Entre os 12 doentes que tomaram oxamniquine houve um que não teve redução da carga parasitária, e nos 11 restantes, esta caiu em média de 291 para 68 ovos por grama de fezes (Tabela 3), dando uma redução da carga parasitária de $76,6 \%$.

Tabela 3 - Redução da carga parasitária em 20 pacientes com esquistossomose tratados com praziquantel ou oxamniquine e não curados

\begin{tabular}{lcccc}
\hline Medicamento & No de doentes & \multicolumn{2}{c}{$N^{\circ}$ de ovos de S. mansoni por grama de fezes } \\
\cline { 3 - 4 } & & Antes do & Depois do & Redução \\
& & tratamento & tratamento \\
\hline Praziquantel & 9 & 281 & 14 & $95,1 \%$ \\
Oxamniquine & $1^{*}$ & 291 & 68 & $76,6 \%$ \\
\hline
\end{tabular}

$\left(^{*}\right)$ Excluido um paciente em que não houve redução de carga parasitária.

A tolerância aos medicamentos foi avaliada em 119 pacientes, tendo sido registradas manifestações de toxicidade ou efeitos colaterais em 61 deles. Destes, $25(40,9 \%)$ haviam tomado oxamniquine e $36(59 \%)$ haviam tomado praziquantel. Em ambos os grupos, as manifestações de intolerância foram consideradas como sendo leves ou moderadas. No grupo tratado com oxamniquine as manifestações mais freqüentes foram, em ordem de freqüência: tonturas, dor abdominal, sonolência e as- 
Emanuel A, Prata A. Comparação entre praziquantel examniquine no tratamento da esquistossomose mansoni. Revista da Sociedade Brasileira de Medicina Tropical 16:90-93, Abr-Jun, 1983

tenia. E no grupo tratado com praziquantel, foram: dor abdominal, tontura, astenia, diarréia, nauseas e cefaléia (Tabela 4).

Tabela 4 - Efeitos colaterais em 119 pacientes com esquistossomose tratados com praziquantel e oxamniquine

\begin{tabular}{lrr} 
Efeitos Colaterais & Praziquantel & Oxamniquine \\
\hline Dor abdominal & $18(30,0 \%)$ & $6(10,2 \%)$ \\
Tontura & $9(15,0 \%)$ & $12(20,3 \%)$ \\
Astenia & $7(11,7 \%)$ & $5(8,5 \%)$ \\
Náusea & $6(10,0 \%)$ & $4(6,8 \%)$ \\
Diarréia & $6(10,0 \%)$ & $3(5,1 \%)$ \\
Cefaléia & $6(10,0 \%)$ & $3(5,1 \%)$ \\
Sonolência & $4(6,7 \%)$ & $5(8,5 \%)$ \\
Febre & $4(6,7 \%)$ & 0 \\
Anorexia & $4(6,7 \%)$ & 0 \\
Sialorréia & $3(5,0 \%)$ & $1(1,7 \%)$ \\
Turvaçào visual & 0 & $3(5,1 \%)$ \\
\hline
\end{tabular}

\section{DISCUSSĀO}

A oxamniquine na dose de $15 \mathrm{mg}$ por quilo de peso corporal mostrou praticamente a mesma percentagem de cura que o praziquantel, em cápsulas, na dose de $55 \mathrm{mg}$ por quilo de peso corporal. Resultados semelhantes foram obtidos por Berti e

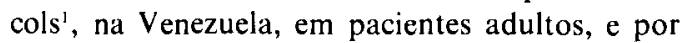
Katz e Rocha' ${ }^{10}$, ao tratarem crianças de 8 a 14 anos, com doses ligeiramente superiores às que empregamos em nossos pacientes. Branchini e cols' também concluiram que não houve superioridade significativa entre as duas drogas, embora obtivessem percentagens de cura um pouco menores que as por nós obtida. Todavia, as doses que eles empregaram foram menores que as utilizadas neste trabalho. A carga parasitária, a julgar pelo número de ovos por grama de fezes, foi acentualmente reduzida com ambas as drogas $(23,4 \%)$.

Ambas as drogas foram relativamente bem toleradas. Embora a metade dos pacientes tenha referido algum efeito colateral, estes geralmente foram de pouca intensidade. A tolerância ao praziquantel em cápsulas, neste trabalho, nos pareceu melhor do que aquela por nós obtida em comprimidos, em estudo anterior ił. Há necessidade de se avaliar a tolerância do prazinquatel nos pacientes com esquistossomose associada à neurocisticercose.

\section{SUMMARY}

We have treated 120 patients between 17 and 19 years old with intestinal or hepatointestinal forms of Schistosoma mansoni infection. Patients were matched in pairs according to the number of eggs per gram of faeces. One member was given oxamniquine in a single dose of $15 \mathrm{mg} / \mathrm{kilo} /$ body weight, and the other praziquantel in a dose of $55 \mathrm{mg} / \mathrm{kilo} /$ of body weight, both in capsule form.

Of the 106 patients adequately evaluated (at least two stool examinations after treatment) 44 (83\%) praziquantel group and 41 (77,3\%) of the oxamniquine group had no eggs in the faeces. Both drugs were well tolerated but oxamniquine appeared slightly better in this regard.

Key words: Schistosomiasis mansoni. Treatment. Oxamniquine. Praziquantel.

\section{REFERENCIAS BIBLIOGRÁFICAS}

1. Berti JJ, Molina BP, Dommerque FS. Tratamiento de la esquistossomose mansoni. Estudio comparativo entre el praziquantel y el oxamniquine. XV Congresso da Sociedade Brasileira de Medicina Tropical, Campinas SP 138:1979

2. Bina JC, Prata A. Oxamniquine in the treatment of schistosomiasis in a population in area with low endemicity. Revista do Instituto de Medicina Tropical de São Paulo 22: 94-97, 1980

3. Branchini MLM, Pedro RJ, Dias LCC, Deberaldini ER. Double-blind clinical trial comparing praziquantel with oxamniquine in the treatment of patients with schistosomiasis mansoni. Revista do Instituto de Medicina Tropical de São Paulo 24:315321, 1982

4. Camargo $\mathrm{S}$. The role of chemotherapy in the special program for control of schistosomiasis. Revista do Instituto de Medicina Tropical de Sào Paulo 22: 98 104, 1980

5. Coura JR, Argento CA, Conceição MJ, Lewis EM, Santos ML, Magalhães P. Field experiences with oral oxamniquine in the treatment of schistosomiasis mansoni. Revista do Instituto de Medicina Tropical de São Paulo 22:77-84, 1980

6. Emanuel A, Prata A. Tratamento da esquistossomose em crianças com praziquantel. Revista do Instituto de Medicina Tropical de São Paulo 4:178-181, 1983.

7. Katz N, Chaves A, Pellegrino J. A simple device for quantitative stoll thick-smear technique in Schistosomiasis mansoni. Revista do Instituto de Medicina Tropical de São Paulo 14: 397-400, 1972 
Emanuel A, Prata A. Comparação entre praziquantel e oxamniquine no tratamento da esquistossomose mansoni. Revista da Sociedade Brasileira de Medicina Tropical 16:90-93, Abr-Jun, 1983

8. Katz N, Grimabaum E, Chaves A, Zicker F, Pellegrino J. Clinical trials with oxamniquine by oral route in Schistosomiasis mansoni. Revista do Instituto de Medicina Tropical de São Paulo 18: 371-377, 1976

9. Katz N, Rocha RS, Chaves A. Preliminary trials with praziquantel in human infections due to $S$. mansoni. Bulletin of the World Health Organization 57: $781-785,1979$

10. Katz N, Rocha RS. Double-blind clinical trial comparing praziquantel with oxamniquine in Schistosomiasis mansoni. Revista do Instituto de Medicina Tropical de São Paulo 24: 310-314, 1982

11. Machado PA. Brazilian program for schistosomiasis control, 1975-1979. American Journal of Tropical Medicine and Hygiene 31: 76-86, 1982
12. Prata A, Lauria L, Figueiredo JFM, Senna PG. Tratamento da esquistossomose mansoni pela oxamniquine em dose única, pela via oral. Revista da Sociedade Brasileira de Medicina Tropical 10: 127-136, 1976

13. Prata A, Castro CN, Silva AE, Paiva M, Macêdo V, Junqueira Jr. LF. Praziquantel no tratamento da esquistossomose mansoni. Revista do Instituto de Medicina Tropical de São Paulo 24: 95-103, 1982

14. Silva AE, Rocha L, Castro CN, Prata A. Tentativa de controle da esquistossomose em uma área endêmica através de quatro tratamentos em massa. Revista do Instituto de Medicina Tropical de São Paulo 23: $260-263,1982$ 